\title{
Quasirelativistic quasilocal finite wave-function collapse model
}

\author{
Philip Pearle* \\ Department of Physics, Hamilton College, Clinton, NY 13323
}

(Dated: November 14, 2018)

\begin{abstract}
A Markovian wave function collapse model is presented where the collapse-inducing operator, constructed from quantum fields, is a manifestly covariant generalization of the mass density operator utilized in the nonrelativistic Continuous Spontaneous Localization (CSL) wave function collapse model. However, the model is not Lorentz invariant because two such operators do not commute at spacelike separation, i.e., the time-ordering operation in one Lorentz frame, the "preferred" frame, is not the time-ordering operation in another frame. However, the characteristic spacelike distance over which the commutator decays is the particle's Compton wavelength so, since the commutator rapidly gets quite small, the model is "almost" relativistic. This "QRCSL" model is completely finite: unlike previous, relativistic, models, it has no (infinite) energy production from the vacuum state. QRCSL calculations are given of the collapse rate for a single free particle in a superposition of spatially separated packets, and of the energy production rate for any number of free particles: these reduce to the CSL rates if the particle's Compton wavelength is small compared to the model's distance parameter. One motivation for QRCSL is the realization that previous relativistic models entail excitation of nuclear states which exceeds that of experiment, whereas QRCSL does not: an example is given involving quadrupole excitation of the ${ }^{74} \mathrm{Ge}$ nucleus.
\end{abstract}

PACS numbers: 03.65.Ta,02.50.Ey

\section{INTRODUCTION}

The Continuous Spontaneous Localization (CSL) model [1, 2] is, currently, the only modification of Schrödinger's equation which satisfactorily describes both standard quantum physics and the macroscopic world we see around us. When a superposition of macroscopic spatially distinguishable states appears, the wave function dynamically undergoes rapid collapse toward one such state.

Since the world is locally Lorentz invariant, it is natural to try to make a relativistic collapse model. I have been working at this for over a decade and, although the models constructed have certain interesting features, each has certain interesting flaws.

The major problem with the first models $[\underline{3},[4,5]$ is that, even to lowest order in the collapse rate parameter $\lambda$, there is an infinite energy production rate per volume from the vacuum. The reason is as follows. In nonrelativistic CSL, a randomly fluctuating classical field $w(\mathbf{x}, t)$ interacts with the mass-density operator (coupling constant $\lambda$ ). Collapse narrows wavepackets, resulting in a small rate of energy increase of particles (the energy is supplied by $w(\mathbf{x}, t)[\underline{6}])$ which is, at present, below experimental observation []. However, in these relativistic models, each vacuum energy-momentum mode, which may be regarded as the ground state of a harmonic oscillator, is likewise narrowed by the collapse interaction. Thus each vacuum mode is no longer just the ground state but is a superposition of ground and excited states, i.e., there is a small rate of creation of particles with that energy-momentum. Since there are an infinite number of modes, than infinite energy production rate per volume of particles from the vacuum.

In nonrelativistic CSL and in these first relativistic models, $w(\mathbf{x}, t)$ is white gaussian noise, i.e., it contains all wavelengths and frequencies in equal amounts. In standard quantum theory, interaction of an operator with classical noise of frequency $\omega$ results in excitation of the quantum system with energy change $\hbar \omega$. That is also the case for collapse models.

I therefore considered models where the noise is non-white gaussian [8, 9] (which entails a non-Markovian state vector evolution) and showed that, indeed, suppression of the frequency $\omega$ in the inverse of the spectrum of $w(\mathbf{x}, t)$ suppresses energy $\hbar \omega$ excitation. This led to a relativisitic model with a tachyonic (momentum-energy relation $\mathbf{k}^{2}-k^{02}=-\mu^{2}$ ) inverse noise spectrum [10], which does not excite the vacuum to lowest order in $\lambda$ since there is no mode of the vacuum which has tachyonic energy-momentum.

However, to order $\lambda^{2}$, once again the ugly spectre(um) of infinite energy production from the vacuum appears. The culprit is that, in this order, there is an internal particle line in the Feynman diagram describing vacuum excitation.

*Electronic address: ppearle@hamilton.edu 
The associated particle propagator, $\left(\mathbf{k}^{2}-k^{02}+M^{2}-i \epsilon\right)^{-1}$, like the white noise spectrum, possesses all wavelengths and energies, which it uses to convert the tachyonic energy-momentum to vacuum production of a pair of particles.

If the particle propagator were on-shell, $\delta\left(\mathbf{k}^{2}-k^{02}+M^{2}\right)$, then the vacuum excitation disappears to this and all orders. This may be achieved by removing the time-ordering operation from the state-vector evolution operator, resulting in a finite relativistically invariant collapse model (RCSL), but at a cost. First, the model is expressed in the interaction picture: with time-ordering one can reconstruct the Schrödinger picture (multiply the interaction picture state vector by $\exp -i H t$, where $H$ is the Hamiltonian) but, without time-ordering, this connection is severed. Second, the model is nonlocal in an unusual sense since, with time ordering, each Feynman diagram describes a series of forward-in-time evolutions but, without time-ordering, these alternate with backward-in-time evolutions. While the time-ordering evolution keeps an evolving particle rigorously within the light cone of its initial spatial state, the non-time-ordering evolution does allow the particle to go out of the light cone in high enough order, albeit with a small probability. Third, and most decisively, as shown in Section $\mathrm{V}$ and Appendix A, to lowest order in $\lambda$ (where time-ordering plays no role), the tachyonic-based theory predicts too great an excitation of nuclear states: the "spontaneous" quadrupole excitation from the ground state $\left(0^{+}\right)$of ${ }^{74}$ Ge to the first excited state $\left(2^{+}\right)$at $.596 \mathrm{MeV}$ greatly exceeds the experimentally observed rate. To my knowledge, this is the first situation where experiment has ruled out a collapse model.

This paper takes another tack. I have been unable to to construct a collapse model simultaneously satisfying 1) relativistic invariance, 2) locality, 3) Markovian evolution, 4) no vacuum excitation, 5) consistency with experiment. Nonrelativistic CSL satisfies all but 1), replacing it with galilean invariance. Relativistic collapse models in refs 3, 4, 5] do not satisfy 4), 5) and RCSL does not satisfy 2), 3) and 5). (See references [11, 12] for a similar assessment of recent interesting models by Rimini and Nicrosini and by Tumulka.) This paper contains a model which satisfies 3), 4), 5), which satisfies 1) and 2) approximately, and which reduces to CSL in the nonrelativistic limit.

\section{NONRELATIVISTIC CSL}

Nonrelativistic CSL is characterized by two equations, the state vector evolution equation and the probability rule.

The evolution equation in the "collapse interaction picture" (where the operators are Heisenberg operators, and the state vector only changes with time due to collapse) is

$$
|\psi, t\rangle_{w}=\mathcal{T} e^{-(4 \lambda)^{-1} \int_{0}^{t} d t d \mathbf{x}[w(\mathbf{x}, t)-2 \lambda A(\mathbf{x}, t)]^{2}}|\psi, 0\rangle
$$

$(\mathcal{T}$ is the time-ordering operator). In Eq.(1), $A(\mathbf{x}, t)$ is an operator essentially proportional to the mass of particles in a spherical volume of radius $a$, and can be written in various ways:

$$
\begin{aligned}
A(\mathbf{x}, 0) & \equiv \sum_{\alpha} \frac{M_{\alpha}}{M}\left(4 \pi a^{2}\right)^{3 / 4} e^{2^{-1} a^{2} \nabla^{2}} \xi_{\alpha}^{\dagger}(\mathbf{x}) \xi_{\alpha}(\mathbf{x}) \\
& =\sum_{\alpha} \frac{M_{\alpha}}{M} \frac{1}{\left(\pi a^{2}\right)^{3 / 4}} \int d \mathbf{b} e^{-\left(2 a^{2}\right)^{-1} \mathbf{b}^{2}} \xi_{\alpha}^{\dagger}(\mathbf{x}+\mathbf{b}) \xi_{\alpha}(\mathbf{x}+\mathbf{b}) \\
& =\sum_{\alpha} \frac{M_{\alpha}}{M}\left(\frac{a^{2}}{4 \pi^{3}}\right)^{3 / 4} \int d \mathbf{p}^{\prime} d \mathbf{p} e^{-2^{-1} a^{2}\left(\mathbf{p}^{\prime}-\mathbf{p}\right)^{2}} e^{-i\left(\mathbf{p}^{\prime}-\mathbf{p}\right) \cdot \mathbf{x}} a_{\alpha}^{\dagger}\left(\mathbf{p}^{\prime}\right) a_{\alpha}(\mathbf{p})
\end{aligned}
$$

where $A(\mathbf{x}, t)=\exp (i H t) A(\mathbf{x}, 0) \exp (-i H t)$, with $H$ the complete Hamiltonian for the interacting particles. In Eqs.(2),

$$
\xi_{\alpha}^{\dagger}(\mathbf{x}) \equiv(2 \pi)^{-3 / 2} \int d \mathbf{p} e^{-i \mathbf{p} \cdot \mathbf{x}} a_{\alpha}^{\dagger}(\mathbf{p})
$$

is the creation operator for a particle of type $\alpha$ (electron, proton, neutron,...) at the position $\mathbf{x}$ (so $\xi_{\alpha}^{\dagger}(\mathbf{x}) \xi_{\alpha}(\mathbf{x})$ is the number density operator for particles of type $\alpha), a_{\alpha}^{\dagger}(\mathbf{p})$ is its momentum $\mathbf{p}$ creation operator, $M_{\alpha}$ is its mass and $M$ is the proton mass. The values of the parameters $\lambda$ and $a$ which characterize the model are generally chosen to be those given in the seminal collapse model of Ghirardi, Rimini and Weber (GRW) [13], $\lambda=10^{-16} \mathrm{sec}^{-1}$ and $a=10^{-5} \mathrm{~cm}$ but there is a range of parameter values allowed by experiment [14].

The probability rule gives the probability density that the classical noise field $\mathrm{w}(\mathbf{x}, \mathrm{t})$ occurs in nature:

$$
\mathcal{P}(w, t) \equiv{ }_{w}\langle\psi, t \mid \psi, t\rangle_{w}
$$

That is, Eq.(1) does not preserve statevector norm, and Eq.(4) says that state vectors of large norm are most likely to occur. It follows from Eqs.(1), (4) that $\int D w \mathcal{P}(w, t)=1$, where $D w \equiv \prod_{\mathbf{x}, t} d w(\mathbf{x}, t)(2 \pi \lambda / d \mathbf{x} d t)^{-1 / 2}$ is the functional 
integration volume element (in doing the integrals, $\mathbf{x}, t$ are discretized, with $w(\mathbf{x}, t)$ regarded as an independent variable for each $(\mathbf{x}, t)$.

It is readily shown that Eqs.(1), (4) entail that a state vector, describing a macroscopic object in a superposition of places, rapidly evolves toward one of the states in the superposition with probability equal to the squared magnitude of its coefficient in the superposition. Essentially, a state which survives when all others have collapsed is one for which the time average of $w(\mathbf{x}, t)$ equals the state's time average of $2 \lambda A(\mathbf{x}, t)$ at each $\mathbf{x}$ (all other behaviors have vanishing probability).

For calculations of physical effects, it is easiest to utilize the density matrix which describes the ensemble of evolutions:

$$
\begin{aligned}
\rho(t) & \equiv \int D w \frac{|\psi, t\rangle_{w w}\langle\psi, t|}{w\langle\psi, t \mid \psi, t\rangle_{w}} \mathcal{P}(w, t) \\
& =\mathcal{T} e^{-(\lambda / 2) \int_{0}^{t} d t d \mathbf{x}\left[A_{L}(\mathbf{x}, t)-A_{R}(\mathbf{x}, t)\right]^{2}} \rho(0),
\end{aligned}
$$

Eq.(5b) follows from putting Eqs.(1), (4) into (5a). In Eq.(5b), when the exponential is expanded in a power series, $A_{L}\left(A_{R}\right)$ operates to the left (right) of $\rho(0)$, and $\mathcal{T}$ time-orders (time-reverse orders) the operators at the left (right).

\section{QUASIRELATIVISTIC MODEL FOR NONINTERACTING PARTICLES}

In what follows, for simplicity, only one type of particle, a "bosonic nucleon" of mass $\mathrm{M}$ shall be considered: the results are trivially extendable to fermions and bosons of many types. The relativistic generalization of the nonrelativistic creation operator $\xi^{\dagger}(\mathbf{x}, \mathbf{t})$ for noninteracting particles (Eq.(3) where exp $i E t$ is stuck into the integral, with $\left.E=\mathbf{p}^{2} / 2 M\right)$ is the negative frequency field operator

$$
\phi^{-}(\mathbf{x}, \mathbf{t}) \equiv(2 \pi)^{-3 / 2} \int d \mathbf{p}(M / E)^{1 / 2} e^{-i p \cdot x} a^{\dagger}(\mathbf{p})
$$

$\left(p \cdot x \equiv \mathbf{p} \cdot \mathbf{x}-E t\right.$, where $\left.E=\left(\mathbf{p}^{2}+M^{2}\right)^{1 / 2}\right)$ which, like the local field $\phi(x)=\phi^{-}(x)+\phi^{+}(x)$, transforms like a scalar under Lorentz transformations. In Eq. (6) we choose the commutation relation $\left[a(\mathbf{p}), a^{\dagger}\left(\mathbf{p}^{\prime}\right)\right]=\delta\left(\mathbf{p}-\mathbf{p}^{\prime}\right)$ so $a(\mathbf{p}) \sqrt{E}$ transforms like a scalar. It is clear that $\phi^{-}(\mathbf{x}, \mathbf{t})$ reduces to $\xi^{\dagger}(\mathbf{x}, \mathbf{t})$ in the nonrelativistic $(c \rightarrow \infty)$ limit $($ except for an additional factor $\exp i M t$, which cancels out in $\left.\phi^{-} \phi^{+}\right)$.

In this quasirelativisitic CSL (QRCSL) model, the evolution equations are also (1) for the state vector, (5) for the density matrix, and (3) for the probability rule. But, for QRCSL, $A(\mathbf{x}, \mathbf{t})$ is defined using an approach from nonlocal relativistic quantum field theory [15], and can be written in various ways parallel to Eqs.(2):

$$
\begin{aligned}
A(x) & \equiv\left(4 \pi a^{2}\right)^{3 / 4} e^{2^{-1} a^{2} \square} \phi^{-}(x) \phi^{+}(x) \\
& =\frac{1}{2^{1 / 2}\left(\pi a^{2}\right)^{5 / 4}} \int d \mathbf{b} d b_{0} e^{-\left(2 a^{2}\right)^{-1}\left[\mathbf{b}^{2}+b_{0}^{2}\right]} \phi^{-}\left(\mathbf{x}+\mathbf{b}, t+i b_{0}\right) \phi^{+}\left(\mathbf{x}+\mathbf{b}, t+i b_{0}\right) \\
& =\left(\frac{a^{2}}{4 \pi^{3}}\right)^{3 / 4} \int d \mathbf{p}^{\prime} d \mathbf{p} \frac{M}{\sqrt{E E^{\prime}}} e^{-2^{-1}\left(p^{\prime}-p\right)^{2} a^{2}} e^{-i\left(p^{\prime}-p\right) \cdot x} a^{\dagger}\left(\mathbf{p}^{\prime}\right) a(\mathbf{p})
\end{aligned}
$$

$\left(\square \equiv \nabla^{2}-\partial_{t}^{2}\right)$

It is apparent from Eqs. (7a,c) that $A(x)$ is a Lorentz scalar. It is also worth noting that the exponent $\left(p^{\prime}-p\right)^{2}$ in $(7 \mathrm{c})$ is spacelike (i.e., positive: in the reference frame where $\left.\mathbf{p}^{\prime}=0,\left(p^{\prime}-p\right)^{2}=2 M(E-M)\right)$. It is easy to see from Eqs.(7) that, in the $c \rightarrow \infty$ limit, $\mathrm{A}(\mathrm{x})$ reduces to the nonrelativistic $A(\mathbf{x}, t)$ (Eqs.(2) with time behavior added).

The equal time commutator, utilizing Eq.(7b), is

$$
\begin{gathered}
{\left[A(\mathbf{x}, t), A\left(\mathbf{x}^{\prime}, t\right)\right]=\left(2 \pi^{5 / 2} a^{5}\right)^{-1} \int d \mathbf{b} d b_{0} d \mathbf{b}^{\prime} d b_{0}^{\prime} e^{-\left(2 a^{2}\right)^{-1}\left[\mathbf{b}^{2}+b_{0}^{2}\right]} e^{-\left(2 a^{2}\right)^{-1}\left[\mathbf{b}^{\prime 2}+b_{0}^{\prime 2}\right]}} \\
{\left[\phi^{-}\left(\mathbf{x}+\mathbf{b}, t+i b_{0}\right) \phi^{+}\left(\mathbf{x}^{\prime}+\mathbf{b}^{\prime}, t+i b_{0}^{\prime}\right)-\phi^{-}\left(\mathbf{x}^{\prime}+\mathbf{b}^{\prime}, t+i b_{0}^{\prime}\right) \phi^{+}\left(\mathbf{x}+\mathbf{b}, t+i b_{0}\right)\right]} \\
{\left[\phi^{+}\left(\mathbf{x}+\mathbf{b}, t+i b_{0}\right), \phi^{-}\left(\mathbf{x}^{\prime}+\mathbf{b}^{\prime}, t+i b_{0}^{\prime}\right)\right]}
\end{gathered}
$$

with

$$
\left[\phi^{+}(x), \phi^{-}\left(x^{\prime}\right)\right]=\frac{M}{(2 \pi)^{3}} \int \frac{d \mathbf{p}}{E} e^{i p \cdot\left(x-x^{\prime}\right)} .
$$


Eq. (9), with the arguments appropriate to (8), is

$$
\left[\phi^{+}\left(\mathbf{x}+\mathbf{b}, t+i b_{0}\right), \phi^{-}\left(\mathbf{x}^{\prime}+\mathbf{b}^{\prime}, t+i b_{0}^{\prime}\right)\right]=\frac{M^{2} K_{1}\left[M \sqrt{\left(\mathbf{x}+\mathbf{b}-\mathbf{x}^{\prime}-\mathbf{b}^{\prime}\right)^{2}+\left(b_{0}-b_{0}^{\prime}\right)^{2}}\right]}{2 \pi^{2} \sqrt{\left(\mathbf{x}+\mathbf{b}-\mathbf{x}^{\prime}-\mathbf{b}^{\prime}\right)^{2}+\left(b_{0}-b_{0}^{\prime}\right)^{2}}} .
$$

where $K_{1}$ is the Bessel Function. Now, $\mathbf{b}, \mathbf{b}^{\prime}, b_{0}, b_{0}^{\prime}$ in Eq.(8) have gaussian distributions with spread $a$. For most of their volume of integration where the gaussians are large, the argument of $K_{1}$ in (10) is quite large, of order $M a=10^{-5} \mathrm{~cm} / 10^{-14} \mathrm{~cm}=10^{9}$. Since for large argument, $K_{1}(z) \rightarrow(\pi / 2 z)^{1 / 2} \mathrm{exp}-z$, in Eq.(8) where the gaussians are large, the factor $K_{1}$ is, for the most part, quite small. It is in this sense that the commutator (8) "almost" vanishes, making the time-ordering operation "almost" frame-independent and the model "quasi"-relativistic.

In calculations of the density matrix using Feynman diagrams, internal particle lines are represented by

$$
\begin{aligned}
\left\langle 0\left|\mathcal{T}\left[\phi^{+}(x), \phi^{-}\left(x^{\prime}\right)\right]\right| 0\right\rangle & =\Theta\left(t-t^{\prime}\right) \frac{M}{(2 \pi)^{3}} \int \frac{d \mathbf{p}}{E} e^{i p \cdot\left(x-x^{\prime}\right)} \\
& =\frac{M i}{(2 \pi)^{4}} \int \frac{d^{4} p}{E} \frac{1}{p^{0}-E+i \epsilon} e^{i p \cdot\left(x-x^{\prime}\right)} \\
& =\frac{2 M i}{(2 \pi)^{4}} \int d^{4} p \frac{1+\left[\left(p^{0}-E\right) / 2 E\right]}{p^{02}-\mathbf{p}^{2}-m^{2}+i \epsilon} e^{i p \cdot\left(x-x^{\prime}\right)} .
\end{aligned}
$$

Eq.(11a) follows from Eq.(9): in (11a), $p=(\mathbf{p}, E)$ while in $(11 \mathrm{~b}, \mathrm{c}), p=\left(\mathbf{p}, p^{0}\right)$.

Eqs.(11) show the lack of Lorentz invariance. However, with $\left(\mathbf{x}-\mathbf{x}^{\prime}, t-t^{\prime}\right)$ replaced by $\left(\mathbf{x}+\mathbf{b}-\mathbf{x}^{\prime}-\mathbf{b}^{\prime}, t+i b_{0}-t^{\prime}-i b_{0}^{\prime}\right)$ in (11a), the integrands of $(11 \mathrm{a}, \mathrm{b}, \mathrm{c})$ acquire the factor $\exp i\left[\mathbf{p} \cdot\left(\mathbf{b}-\mathbf{b}^{\prime}\right)+E\left(b_{0}-b_{0}^{\prime}\right)\right]$ : for spacelike $\left(x-x^{\prime}\right)$, the propagator equals $\Theta\left(t-t^{\prime}\right)$ multiplied by Eq.(10) with argument $\left.\left[M\left(\mathbf{x}+\mathbf{b}-\mathbf{x}^{\prime}-\mathbf{b}^{\prime}\right)^{2}-\left(t+i b_{0}-t^{\prime}-i b_{0}^{\prime}\right)^{2}\right]^{1 / 2}\right)$, and the previous discussion of how this "almost" vanishes applies.

One might consider writing the propagator in each Feynman diagram, and thus the whole density matrix, as the sum of a relativistic piece plus a non-relativistic correction. There are various ways to achieve this. One might split the propagator in space-time into its expression within the forward light-cone and zero elsewhere, with the correction as the spacelike remainder. Eq.(11c) shows another split, in momentum space, with the relativisitic part equal to the usual propagator (recall that our definition (6) of $\phi^{-}$is $(2 M)^{1 / 2}$ times the usual definition) plus a part which is relatively small for $p^{0} \approx E$.

\section{CALCUlations}

The density matrix evolution equation and its perturbation series follow from Eq.(5b):

$$
\begin{aligned}
\frac{d \rho(t)}{d t} & =-(\lambda / 2) \int d \mathbf{x}[A(\mathbf{x}, t),[A(\mathbf{x}, t), \rho(t)]] \\
\rho(t) & =\sum_{n=0}^{\infty} \frac{(\lambda / 2)^{n}}{n !} \int_{0}^{t} d x_{n} \ldots \int_{0}^{t} d x_{1} \mathcal{T}\left[A\left(x_{n}\right), \ldots\left[A\left(x_{1}\right), \rho(t)\right] \ldots\right] .
\end{aligned}
$$

In this section, Eq.(12a) is used to calculate the rate for the wave function of a single free particle, in a superposition of two widely separated packets, to collapse to one of the packets. Next, the rate of energy increase for $N$ free particles is found. The following section discusses the formalism of QRCSL when particles are interacting. Finally, this result is used to calculate, to first order in $\lambda$, the quadrupole excitation rate of the ${ }^{74} \mathrm{Ge}$ nucleus from its ground state to its first excited state. The result is compared with the present experimental upper limit on the rate of "spontaneous" excitation in Ge.

\section{A. Collapse Rate for a Single Free Particle}

Consider a single particle initially in a superposition $|\psi, 0\rangle=\alpha|L\rangle+\beta|R\rangle$, where $|L\rangle$ and $|R\rangle$ are widely separated wavepackets, so far apart that, to high accuracy, their regions of support do not overlap over the time interval $t$. Let $\left|\mathbf{x}_{L}\right\rangle\left(\left|\mathbf{x}_{R}\right\rangle\right)$ be a position eigenstate within the left (right) region of support. The off-diagonal element of the density 
matrix, using Eqs.(12a) and (7c), satisfies:

$$
\begin{aligned}
\frac{d\left\langle\mathbf{x}_{L}|\rho(t)| \mathbf{x}_{R}\right\rangle}{d t}= & -(\lambda / 2)\left(\frac{a^{2}}{4 \pi^{3}}\right)^{3 / 2} M^{2} \int d \mathbf{x} \int \frac{d \mathbf{p}_{1}}{\sqrt{E_{1}}} \frac{d \mathbf{p}_{2}}{\sqrt{E_{2}}} \frac{d \mathbf{p}_{3}}{\sqrt{E_{3}}} \frac{d \mathbf{p}_{4}}{\sqrt{E_{4}}} \\
\cdot & e^{-2^{-1} a^{2}\left[\left(p_{1}-\left(p_{2}\right)^{2}+\left(p_{3}-\left(p_{4}\right)^{2}\right]\right.\right.} e^{-i\left(p_{1}-p_{2}+p_{3}-p_{4}\right) \cdot x} \\
\cdot & \left\{\delta\left(\mathbf{p}_{2}-\mathbf{p}_{3}\right) \frac{1}{(2 \pi)^{3}} \int d \mathbf{z}\left[e^{i \mathbf{p}_{1} \cdot \mathbf{x}_{L}-i \mathbf{p}_{4} \cdot \mathbf{z}}\left\langle\mathbf{z}|\rho(t)| \mathbf{x}_{R}\right\rangle+e^{i \mathbf{p}_{1} \cdot \mathbf{z}-i \mathbf{p}_{4} \cdot \mathbf{x}_{R}}\left\langle\mathbf{x}_{L}|\rho(t)| \mathbf{z}\right\rangle\right]\right. \\
& \left.-2 \frac{1}{(2 \pi)^{6}} \int d \mathbf{z} \int d \mathbf{z}^{\prime} e^{i \mathbf{p}_{1} \cdot \mathbf{x}_{L}-i \mathbf{p}_{2} \cdot \mathbf{z}+i \mathbf{p}_{3} \cdot \mathbf{z}^{\prime}-i \mathbf{p}_{4} \cdot \mathbf{x}_{R}}\left\langle\mathbf{z}|\rho(t)| \mathbf{z}^{\prime}\right\rangle\right\} .
\end{aligned}
$$

First, it can be seen that the last term in the curly brackets of Eq.(13) (arising from the term $-\lambda \int d \mathbf{x} A(\mathbf{x}, t) \rho(t) A(\mathbf{x}, t)$ in Eq. (12a)) may be neglected. Consider the integral over $\mathbf{p}_{1}$, $\mathbf{p}_{2}$ which appears in this term:

$$
\begin{aligned}
f\left(\mathbf{x}_{L}, \mathbf{x}, \mathbf{z} ; t\right) & \equiv \int \frac{d \mathbf{p}_{1}}{\sqrt{E_{1}}} \frac{d \mathbf{p}_{2}}{\sqrt{E_{2}}} e^{-2^{-1} a^{2}\left(p_{1}-p_{2}\right)^{2}} e^{-i\left(p_{1}-p_{2}+p_{3}-p_{4}\right) \cdot x} e^{i \mathbf{p}_{1} \cdot \mathbf{x}_{L}-i \mathbf{p}_{2} \cdot \mathbf{z}} \\
& \approx \sim e^{-\left(2 a^{2}\right)^{-1}\left(\mathbf{x}-\mathbf{x}_{L}\right)^{2}} \delta\left(\mathbf{z}-\mathbf{x}_{L}\right)
\end{aligned}
$$

where $\approx$ in $(14 \mathrm{~b})$ means that we have set $t=0$ and $E_{1}=E_{2}=M$. Even without the approximation, for any $t$, if any one argument $(\mathbf{x}$ or $\mathbf{z})$ of $f$ lies in $L$ and another in $R$, then $f \approx 0$. Now, the term under consideration has the form

$$
\begin{aligned}
& \sim \int d \mathbf{x} d \mathbf{z} d \mathbf{z}^{\prime} f\left(\mathbf{x}_{L}, \mathbf{x}, \mathbf{z} ; t\right) f^{*}\left(\mathbf{x}_{R}, \mathbf{x}, \mathbf{z}^{\prime} ; t\right)\left\langle\mathbf{z}|\rho(t)| \mathbf{z}^{\prime}\right\rangle \\
& =\int d \mathbf{x} d \mathbf{z} d \mathbf{z}^{\prime} f\left(\mathbf{x}_{L}, \mathbf{x}, \mathbf{z}_{L} ; t\right) f^{*}\left(\mathbf{x}_{R}, \mathbf{x}, \mathbf{z}_{R}^{\prime} ; t\right)\left\langle\mathbf{z}_{L}|\rho(t)| \mathbf{z}_{R}^{\prime}\right\rangle \approx 0 .
\end{aligned}
$$

In (15b), $\mathbf{z}\left(\mathbf{z}^{\prime}\right)$ is restricted to $\mathbf{z}_{L}\left(\mathbf{z}_{R}^{\prime}\right)$ since, otherwise, $f\left(f^{*}\right)$ would vanish, and the result vanishes because either $f$ or $f^{*}$ vanishes for every $\mathbf{x}$. in

In the remaining terms of (13), first perform the integral over $\mathbf{x}$, followed by the integrals over $p_{3}, p_{4}$, which results

$$
\begin{aligned}
\frac{d\left\langle\mathbf{x}_{L}|\rho(t)| \mathbf{x}_{R}\right\rangle}{d t}= & -(\lambda / 2)\left(\frac{a^{2}}{4 \pi^{3}}\right)^{3 / 2} M^{2} \int \frac{d \mathbf{p}_{1}}{E_{1}} \frac{d \mathbf{p}_{2}}{E_{2}} e^{-a^{2}\left(p_{1}-p_{2}\right)^{2}} \\
& \int d \mathbf{z}\left[e^{i \mathbf{p}_{1} \cdot\left(\mathbf{x}_{L}-\mathbf{z}\right)}\left\langle\mathbf{z}|\rho(t)| \mathbf{x}_{R}\right\rangle+e^{-i \mathbf{p}_{1} \cdot\left(\mathbf{x}_{R}-\mathbf{z}\right)}\left\langle\mathbf{x}_{L}|\rho(t)| \mathbf{z}\right\rangle\right] .
\end{aligned}
$$

Next, the integral over $\mathbf{p}_{2}$ can be performed:

$$
\int \frac{d \mathbf{p}_{2}}{E_{2}} e^{-a^{2}\left(p_{1}-p_{2}\right)^{2}}=\left(2 \pi / a^{2}\right) e^{2 a^{2} M^{2}} K_{1}\left(2 a^{2} M^{2}\right),
$$

followed by the integral over $\mathbf{p}_{1}$ :

$$
\int \frac{d \mathbf{p}_{1}}{E_{1}} e^{i \mathbf{p}_{1} \cdot\left(\mathbf{x}_{L}-\mathbf{z}\right)}=4 \pi M K_{1}\left(M\left|\mathbf{x}_{L}-\mathbf{z}\right|\right) /\left|\mathbf{x}_{L}-\mathbf{z}\right| .
$$

The evolution equation now reads:

$$
\begin{aligned}
\frac{d\left\langle\mathbf{x}_{L}|\rho(t)| \mathbf{x}_{R}\right\rangle}{d t}= & -\left(\lambda a M^{3} / 2 \pi^{5 / 2}\right) e^{2 a^{2} M^{2}} K_{1}\left(2 a^{2} M^{2}\right) \\
& \cdot \int d \mathbf{z}\left[\frac{K_{1}\left(M\left|\mathbf{x}_{L}-\mathbf{z}\right|\right)}{\left|\mathbf{x}_{L}-\mathbf{z}\right|}\left\langle\mathbf{z}|\rho(t)| \mathbf{x}_{R}\right\rangle+\frac{K_{1}\left(M\left|\mathbf{x}_{R}-\mathbf{z}\right|\right)}{\left|\mathbf{x}_{R}-\mathbf{z}\right|}\left\langle\mathbf{x}_{L}|\rho(t)| \mathbf{z}\right\rangle\right]
\end{aligned}
$$

Eq. (19) is exact.

Now, specialize to the case where $a$ is much larger than the particle Compton wavelength $M^{-1}$ so

$$
e^{2 a^{2} M^{2}} K_{1}\left(2 a^{2} M^{2}\right) \approx(2 a M)^{-1} \pi^{1 / 2} .
$$


Further, if the wave packets change slowly on the distance scale of $M^{-1}$ then, in the integrand of Eq.(19), $K_{1}(M \mid \mathbf{x}-$ $\mathbf{z} \mid) /|\mathbf{x}-\mathbf{z}|$ may be well approximated by a delta-function, whose numerical coefficient may be found by integrating Eq.(18) over $\mathbf{z}$ :

$$
K_{1}(M|\mathbf{x}-\mathbf{z}|) /|\mathbf{x}-\mathbf{z}| \approx\left(2 \pi^{2} / M^{2}\right) \delta(\mathbf{x}-\mathbf{z})
$$

The result of inserting (20), (21) into (19) gives the result:

$$
\frac{d\left\langle\mathbf{x}_{L}|\rho(t)| \mathbf{x}_{R}\right\rangle}{d t}=-\lambda\left\langle\mathbf{x}_{L}|\rho(t)| \mathbf{x}_{R}\right\rangle,
$$

i.e., rate of decay of the off-diagonal density matrix element is $\lambda$, the same rate as for nonrelativistic CSL.

\section{B. Energy Creation Rate For Free Particles}

Calculation of the energy creation rate for $n$ free particles begins by multiplying Eq.(12a) by the free Hamiltonian $H_{0}$ and taking the trace:

$$
\begin{aligned}
\frac{d \bar{H}}{d t} & =-\frac{\lambda}{2} \int d \mathbf{x} \operatorname{Tr}\left\{\left[A(x),\left[A(x), H_{0}\right]\right] \rho(t)\right\} \\
& =-\frac{\lambda i}{2} \int d \mathbf{x} \operatorname{Tr}\{[A(x), \dot{A}(x)] \rho(t)\},
\end{aligned}
$$

where $\bar{H}(t) \equiv \operatorname{Tr}\left\{H_{0} \rho(t)\right\}$. Using the expression (7c) for $A(x)$, the commutator can be evaluated. Then, the integral over $\mathbf{x}$ can be performed and, using the resulting delta-functions of momentum differences, two of the momentum integrals can be performed, resulting in

$$
\begin{aligned}
& \frac{d \bar{H}(t)}{d t}=\frac{\lambda M^{2} a^{3}}{2 \pi^{3 / 2}} \int \frac{d \mathbf{p}_{1}}{E_{1}} \frac{d \mathbf{p}_{2}}{E_{2}}\left(E_{2}-E_{1}\right) e^{-a^{2}\left(p_{1}-p_{2}\right)^{2}} \\
\cdot & \operatorname{Tr}\left\{\left[a^{\dagger}\left(\mathbf{p}_{1}\right) a\left(\mathbf{p}_{1}\right)-a^{\dagger}\left(\mathbf{p}_{2}\right) a\left(\mathbf{p}_{2}\right)\right] \rho(t)\right\} \\
= & \frac{\lambda M^{2} a^{3}}{\pi^{3 / 2}} \operatorname{Tr}\left\{\left[\int \frac{d \mathbf{p}_{1}}{E_{1}} a^{\dagger}\left(\mathbf{p}_{1}\right) a\left(\mathbf{p}_{1}\right) \int d \mathbf{p}_{2} e^{-a^{2}\left(p_{1}-p_{2}\right)^{2}}-N \int \frac{d \mathbf{p}_{2}}{E_{2}} e^{-a^{2}\left(p_{1}-p_{2}\right)^{2}}\right] \rho(t)\right\}
\end{aligned}
$$

In Eq.(24b), $N \equiv \int d \mathbf{p}_{1} a^{\dagger}\left(\mathbf{p}_{1}\right) a\left(\mathbf{p}_{1}\right)$ is the number-of-particles operator.

The last integral in Eq.(24b) over $\mathbf{p}_{2}$ is given in Eq.(17). The first integral over $\mathbf{p}_{2}$ is

$$
\int d \mathbf{p}_{2} e^{-a^{2}\left(p_{1}-p_{2}\right)^{2}}=\left(2 \pi / a^{2}\right) E_{1} e^{2 a^{2} M^{2}}\left[K_{0}\left(2 a^{2} M^{2}\right)+\left(a^{2} M^{2}\right)^{-1} K_{1}\left(2 a^{2} M^{2}\right)\right]
$$

Because (25) is proportional to $E_{1}$, the first integral in Eq. $(24 \mathrm{~b})$ over $\mathbf{p}_{1}$ is $\sim N$. Since $\operatorname{Tr}\{N \rho(t)\}=n \operatorname{Tr} \rho(t)=n$, one obtains the exact result that $\bar{H}(t)$ increases linearly with time:

$$
\frac{d \bar{H}(t)}{d t}=\lambda n M^{2} a 2 \pi^{-1 / 2} e^{2 a^{2} M^{2}}\left[K_{0}\left(2 a^{2} M^{2}\right)-K_{1}\left(2 a^{2} M^{2}\right)\left(1-\left(a^{2} M^{2}\right)^{-1}\right)\right] .
$$

In the case where $a M>>1$, using the series expansions

$$
K_{0}(z)=\sqrt{\frac{\pi}{2 z}} e^{-z}\left[1-\frac{1}{8 z}\right], \quad K_{1}(z)=\sqrt{\frac{\pi}{2 z}} e^{-z}\left[1+\frac{3}{8 z}\right],
$$

Eq.(26) becomes:

$$
\frac{d \bar{H}(t)}{d t}=\frac{3 \lambda n}{4 M a^{2}}
$$

the same energy creation rate as for nonrelativistic CSL. 


\section{QRCSL FOR INTERACTING PARTICLES}

The derivative of Eq.(1),

$$
d|\psi, t\rangle_{w} / d t=-(4 \lambda)^{-1} \int d \mathbf{x}[w(x)-2 \lambda A(x)]^{2}|\psi, t\rangle_{w}
$$

where $A(x)$ is given by Eqs.(7), is the QRCSL evolution equation for noninteracting particles in the "collapse interaction picture," where the field operators evolve freely and the state vector changes only due to the collapse evolution. As usual, the Schrd̈inger picture statevector is $|\psi, t\rangle_{w}^{s}=\exp -i H_{0} t|\psi, t\rangle_{w}$, and its evolution equation follows from Eq.(29):

$$
d|\psi, t\rangle_{w}^{s} / d t=-i H_{0}|\psi, t\rangle_{w}^{s}-(4 \lambda)^{-1} \int d \mathbf{x}[w(x)-2 \lambda A(\mathbf{x}, 0)]^{2}|\psi, t\rangle_{w}^{s},
$$

where $A(\mathbf{x}, 0)$ is given by Eqs. (7) with $t=0$ (so, in particular, the operators in (7b) still have $i b_{0}$ as time arguments). In the Schrödinger picture, the operators do not evolve, and the state vector changes with time due to the free evolution as well as due to the collapse evolution.

As usual, to add interaction, one replaces $H_{0}$ by $H=H_{0}+V$ in Eq.(30). Transforming back to the interaction picture gives:

$$
d|\psi, t\rangle_{w} / d t=-i V(t)|\psi, t\rangle_{w}-(4 \lambda)^{-1} \int d \mathbf{x}[w(x)-2 \lambda A(x)]^{2}|\psi, t\rangle_{w}
$$

where $V(t) \equiv \exp \left(i H_{0} t\right) V \exp -\left(i H_{0} t\right)$ is a Lorentz scalar, the four-integral of a local scalar density. However, although Eq.(31) is form covariant, it is not Lorentz invariant because, not only doesn't $A$ commute with itself at space-like separation, it usually will not commute with the local scalar density at space-like separation either. However, such a local density is constructed from $\phi=\phi^{+}+\phi^{-}$, so its commutator with $A$ falls off exponentially with space-like separation as in (10). In this sense this interacting QRCSL model is also "quasi-relativistic." In this interaction picture, the quantum fields evolve freely and the state vector evolves due to the interaction and the collapse.

For some calculations, it is useful to work in the collapse-interaction picture, where the fields are Heisenberg fields, evolving according to the interacting quantum field theory and the state-vector only changes with time due to the collapse evolution. Using (31) to go to the Schrödinger picture and, as usual, defining the collapse-interaction picture statevector as $|\psi, t\rangle_{w}^{\prime}=\exp (i H t)|\psi, t\rangle_{w}^{s}$, one obtains

$$
d|\psi, t\rangle_{w}^{\prime} / d t=-(4 \lambda)^{-1} \int d \mathbf{x}\left[w(x)-2 \lambda A^{\prime}(x)\right]^{2}|\psi, t\rangle_{w}^{\prime}
$$

where $A^{\prime}(x)=\exp (i H t) A(\mathbf{x}, 0) \exp -(i H t)$. It is worth emphasizing that $A^{\prime}(x)$ is not given by Eq.(7b) with operators $\phi^{ \pm}\left(\mathbf{x}+\mathbf{b}, t+i b_{0}\right)$ replaced by $\exp \left[i H\left(t+i b_{0}\right)\right] \phi^{ \pm}(\mathbf{x}+\mathbf{b}, 0) \exp -\left[i H\left(t+i b_{0}\right)\right]$ but, rather, is composed of operators $\exp (i H t) \phi^{ \pm}\left(\mathbf{x}+\mathbf{b}, i b_{0}\right) \exp -(i H t)$, according to this prescription.

\section{A. Bound State Excitation to Lowest Order}

The density matrix evolution equation which follows from Eq.(32) is Eq.(5b) with $A$ replaced by $A^{\prime}$. To lowest order in $\lambda$, this is

$$
\left.\rho(t)=\rho(0)-(\lambda / 2) \int_{0}^{t} d t d \mathbf{x}\left[A^{\prime 2}(\mathbf{x}, t) \rho(0)+\rho(0) A^{\prime 2}(\mathbf{x}, t)-2 A^{\prime}(\mathbf{x}, t)\right) \rho(0) A^{\prime}(\mathbf{x}, t)\right] .
$$

Time ordering plays no role to lowest order, so the expression (33) is Lorentz invariant. Take $\rho(0)=\left|E_{i}\right\rangle\left\langle E_{i}\right|$, where $\left|E_{i}\right\rangle$ is a bound $N$-particle state that is an energy eigenstate with eigenvalue $E_{i}$, and is also an eigenstate with eigenvalue 0 of the center of mass operator $\mathbf{Q} \equiv \sum_{n=1}^{N} \mathbf{X}_{n} / N\left(\mathbf{X}_{n}\right.$ is the position operator of the $n$th particle). It is desired to calculate the probability that the system is excited to the bound energy eigenstate $\left|E_{f}\right\rangle\left(H\left|E_{f}\right\rangle=E_{f}\left|E_{f}\right\rangle\right.$ and $\left.\mathbf{Q}\left|E_{f}\right\rangle=0\right)$. Because $\left\langle E_{i} \mid E_{f}\right\rangle=0$, only the last term in (33) contributes:

$$
\left\langle E_{f}|\rho(t)| E_{f}\right\rangle=\lambda \int_{0}^{t} d t d \mathbf{x}\left\langle E_{f}\left|A^{\prime}(\mathbf{x}, t)\right| E_{i}\right\rangle\left\langle E_{i}\left|A^{\prime}(\mathbf{x}, t)\right| E_{f}\right\rangle
$$


Moreover, since $\left\langle E_{f}\left|A^{\prime}(\mathbf{x}, t)\right| E_{i}\right\rangle=\exp i\left(E_{f}-E_{i}\right)\left\langle E_{f}|A(\mathbf{x}, 0)| E_{i}\right\rangle$, the integrand in (34) is time independent, so the excitation rate $\Gamma \equiv d\left\langle E_{f}|\rho(t)| E_{f}\right\rangle / d t$ is constant:

$$
\Gamma=\lambda \int d \mathbf{x}\left|\left\langle E_{f}|A(\mathbf{x}, 0)| E_{i}\right\rangle\right|^{2} .
$$

At this point, assume that the particles in the initial and final states move nonrelativistically, $(\mathbf{p} / m c)^{2}<<1$, so that, in the expression (7c) for $A(x)$, one can make the approximations $E \approx E^{\prime} \approx M$ and $\left(p-p^{\prime}\right)^{2} \approx\left(\mathbf{p}-\mathbf{p}^{\prime}\right)^{2}$. Then $A(\mathbf{x}, 0)$ in Eq.(35) becomes the non-relativistic expression (2). For completeness, the analysis leading to Eq.(37) shall be given here, instead of just quoted [14, 16]. Start by noting that $\int d \mathbf{x}^{\prime} F\left(\mathbf{x}^{\prime}\right) \xi^{\dagger}\left(\mathbf{x}^{\prime}\right) \xi\left(\mathbf{x}^{\prime}\right)=\sum_{n=1}^{N} F\left(\mathbf{X}_{n}\right)$ where $F$ is an arbitrary function. Use of the form $(2 \mathrm{~b})$ for $A(\mathbf{x}, 0)$ in Eq.(35) results in:

$$
\begin{aligned}
\Gamma & =\lambda\left(\pi a^{2}\right)^{-3 / 2} \int d \mathbf{x}\left|\left\langle E_{f}\left|\int d \mathbf{x}^{\prime} e^{-\left(2 a^{2}\right)^{-1}\left(\mathbf{x}-\mathbf{x}^{\prime}\right)^{2}} \xi^{\dagger}\left(\mathbf{x}^{\prime}\right) \xi\left(\mathbf{x}^{\prime}\right)\right| E_{i}\right\rangle\right|^{2} \\
& =\lambda\left(\pi a^{2}\right)^{-3 / 2} \int d \mathbf{x}\left|\left\langle E_{f}\left|\sum_{n=1}^{N} e^{-\left(2 a^{2}\right)^{-1}\left(\mathbf{x}-\mathbf{X}_{n}\right)^{2}}\right| E_{i}\right\rangle\right|^{2} \\
& =\lambda \sum_{n, m=1}^{N}\left\langle E_{f}\left|\left\{e^{-\left(4 a^{2}\right)^{-1}\left(\mathbf{X}_{n L}-\mathbf{X}_{m R}\right)^{2}}\left|E_{i}\right\rangle\left\langle E_{i}\right|\right\}\right| E_{f}\right\rangle
\end{aligned}
$$

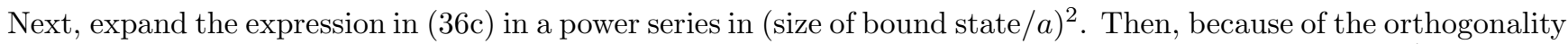
of the initial and final states and because $\mathbf{Q}\left|E_{i, f}\right\rangle=0$, the first nonvanishing term of (36c) is of order $a^{-4}$ :

$$
\Gamma=\lambda(2 a)^{-4}\left[\left|\left\langle E_{f}\left|\sum_{n=1}^{N} \mathbf{X}_{n}^{2}\right| E_{i}\right\rangle\right|^{2}+2 \sum_{n=1, m=1}^{N} \sum_{i, j=1}^{3}\left\langle E_{f}\left|\mathbf{X}_{n}^{i} \mathbf{X}_{n}^{j}\right| E_{i}\right\rangle\left\langle E_{i}\left|\mathbf{X}_{m}^{i} \mathbf{X}_{m}^{j}\right| E_{f} \mid\right\rangle\right] .
$$

\section{B. Excitation of ${ }^{74}$ Ge Nucleus}

Now, Eq.(37) is to be applied to collapse-induced spontaneous excitation of a proton from the ground state $\left(0^{+}\right)$ of a ${ }^{74} \mathrm{Ge}$ nucleus (the largest percentage isotope-36.5\%-in naturally occurring Ge) to its first excited state $\left(2^{+}\right)$ at $.596 \mathrm{MeV}$ [17]. The experimental upper limit on spontaneous emission of .596MeV gammas in Ge, obtained by observing the radiation from an isolated slab of Ge for a long time, is $\approx .03$ counts/kg-day (in a $2 \mathrm{MeV}$ bin) [18]. The analysis for spinless particles of one mass given above can be applied to this case because the proton and neutron have almost the same mass and there is no spin-flip involved in this transition.

One readily finds from (37) the expression for the quadrupole excitation rate:

$$
\Gamma=(\pi / 15)\left(\lambda / a^{4}\right) \sum_{m, m^{\prime}=-2}^{2}\left|\left\langle 2^{+}, m^{\prime}\left|\sum_{n=1}^{Z} \mathbf{X}_{n}^{2} Y_{2 m}\left(\Theta_{n}, \Phi_{n}\right)\right| 0^{+}\right\rangle\right|^{2},
$$

where $\Theta_{n}, \Phi_{n}$ are angle operators for the $n$th particle and $Y_{2 m}$ is a spherical harmonic.

Now, the lifetime $\tau$ of the $2^{+}$state is given by the expression [19]

$$
\tau^{-1}=\left(4 \pi / 3 \cdot 5^{3}\right) c k^{5}\left(e^{2} / \hbar c\right) \sum_{m, m^{\prime}=-2}^{2}\left|\left\langle 2^{+}, m^{\prime}\left|\sum_{n=1}^{Z} \mathbf{X}_{n}^{2} Y_{2 m}\left(\Theta_{n}, \Phi_{n}\right)\right| 0^{+}\right\rangle\right|^{2},
$$

where $k \approx 3.2 \cdot 10^{10} \mathrm{~cm}^{-1}$ is the $.596 \mathrm{MeV}$ photon wavenumber. From Eqs. (38), (39) is obtained

$$
\Gamma_{Q R C S L}=(5 / 2)^{2} \lambda\left[(a k)^{4}\left(e^{2} / \hbar c\right) k c \tau\right]^{-1} \approx 5 \cdot 10^{-16} \text { counts/kg-day, }
$$

with use of the numbers $\tau=17.9 \mathrm{psec}$ as the experimental lifetime of the state, $\approx 8.3 \cdot 10^{24}$ as the number of nucleii $/ \mathrm{kg}$ of Ge (so there are $\approx 3.0 \cdot 10^{24}{ }^{74} \mathrm{Ge}$ nucleii/kg), and $8.6 \cdot 10^{4} \mathrm{sec} /$ day. $\Gamma_{Q R C S L}$ is well below the experimental upper limit of $3 \cdot 10^{-2}$ counts/kg-day.

This contrasts with the situation for the relativistic collapse model RCSL. From Eqs.(A4) and (39) is obtained

$$
\Gamma_{R C S L}=\left(5 / 3 \pi^{2}\right) \lambda a\left[\left(e^{2} / \hbar c\right) c \tau\right]^{-1} \approx 5 \cdot 10^{10} \text { counts/kg-day }
$$


which far exceeds the experimental upper limit. These calculations were performed assuming the GRW values for $\lambda$, $a$ but, for most of the range of these parameters allowed by other considerations [14], $\Gamma_{R C S L}$ is still excessive. The reason for the difference in excitation between QRCSL and RCSL is that, in the former, just as in nonrelativistic CSL, collapse narrows the excited particle's wavefunction to $a$ whereas, in the latter, collapse narrows the wavefunction to $\left[a^{-2}+\left(E_{f}-E_{i}\right)^{2}\right]^{-1 / 2}$.

\section{CONCLUDING REMARKS}

Because all previous CSL-type relativistic collapse models except RCSL are untenable since they produce infinite energy/sec-vol from the vacuum, and RCSL produces too much nuclear excitation, the QRCSL model has been suggested. It has form-invariant equations, but it fails to be relativistic because its Lorentz invariant operators do not commute at space-time separation. However, since these operators "almost" commute, I believe that such quasi-relativistic behavior is worth consideration, as a close and experimentally testable variant of special relativity combined with a description of collapse that is as close as could be expected to nonrelativistic CSL.

However, the model is, after all, described in a preferred frame, the one where the time-ordering operation is defined. One might tentatively identify the preferred frame with the local co-moving frame of the universe [20]. Exploration of the extent of violation of Lorentz invariance for various hypothetical situations is certainly of interest. Since QRCSL's slow speed limit is CSL which, so far, has defied experimental refutation, it may be worthwhile to examine schemes whereby detectors move at high speeds. One may also examine whether the frame dependent, although non-detectable, wave packet collapse locales and times (e.g., in EPR-type situations) in relativistic collapse models are similar to those of QRCSL, or if the preferred frame's wave packet collapse locales and times might, in some sense, be preferred

\section{APPENDIX A: BOUND STATE EXCITATION IN RCSL}

In RCSL, the only finite relativistic collapse model extant, the expression for the excitation probability comparable to Eq.(34) (but here taken in the nonrelativistic limit) is

$$
\left\langle E_{f}|\rho(t)| E_{f}\right\rangle=4 \lambda a \int_{0}^{t} d x d x^{\prime} G\left(x-x^{\prime}\right)\left\langle E_{f}\left|\xi^{\dagger}(x) \xi(x)\right| E_{i}\right\rangle\left\langle E_{i}\left|\xi^{\dagger}\left(x^{\prime}\right) \xi\left(x^{\prime}\right)\right| E_{f}\right\rangle .
$$

In Eq.(A1), $G\left(x-x^{\prime}\right)=(2 \pi)^{-4} \int d p \exp i p \cdot\left(x-x^{\prime}\right) \delta\left(p^{2}-a^{-2}\right)$, i.e., this is a non-Markovian model with a tachyonic noise spectrum whose "tachyon mass" is $a^{-1} \approx 2 \mathrm{eV}$. The operator $\xi^{\dagger}(x)=\exp i H t \xi^{\dagger}(\mathbf{x}, 0) \exp -i H t$, with $\xi^{\dagger}(\mathbf{x}, 0)$ given by Eq.(3), is the Heisenberg creation operator. Use this first, to pull out the time dependence from the matrix elements in (A1), letting $H$ act on the energy eigenstates, and then perform the time integrals with the result

$$
\begin{aligned}
& \left\langle E_{f}|\rho(t)| E_{f}\right\rangle=4(2 \pi)^{-4} \lambda a \int_{0}^{t} d x d x^{\prime} d p e^{i \mathbf{p} \cdot\left(\mathbf{x}-\mathbf{x}^{\prime}\right)} \delta\left(p^{2}-a^{-2}\right) \\
& \quad \cdot\left\{\sin \left[\left(E_{f}-E_{i}-p^{0}\right) t / 2\right] /\left[\left(E_{f}-E_{i}-p^{0}\right) / 2\right]\right\}^{2}\left\langle E_{f}\left|\xi^{\dagger}(\mathbf{x}) \xi(\mathbf{x})\right| E_{i}\right\rangle\left\langle E_{i}\left|\xi^{\dagger}\left(\mathbf{x}^{\prime}\right) \xi\left(\mathbf{x}^{\prime}\right)\right| E_{f}\right\rangle .
\end{aligned}
$$

For large $t,[\sin (\alpha t) / \alpha]^{2} \approx t \pi \delta(\alpha)$. Then, using this delta function to perform the integral over $p^{0}$, and utilizing $\int d \mathbf{x}^{\prime} F\left(\mathbf{x}^{\prime}\right) \xi^{\dagger}\left(\mathbf{x}^{\prime}\right) \xi\left(\mathbf{x}^{\prime}\right)=\sum_{n=1}^{N} F\left(\mathbf{X}_{n}\right)$ as was done in obtaining Eq.(36), we obtain

$$
\begin{gathered}
\Gamma=\lambda a 2^{-1} \pi^{-3} \int d \mathbf{p} \delta\left[\mathbf{p}^{2}-\left(E_{f}-E_{i}\right)^{2}-a^{-2}\right] \sum_{n, m=1}^{N}\left\langle E_{f}\left|\left\{e^{i \mathbf{p} \cdot\left(\mathbf{X}_{n L}-\mathbf{X}_{m R}\right)}\left|E_{i}\right\rangle\left\langle E_{i}\right|\right\}\right| E_{f}\right\rangle \\
=\lambda a \pi^{-2} \sum_{n, m=1}^{N}\left\langle E_{f}\left|\left\{\left[\sin \left(k\left|\mathbf{X}_{n L}-\mathbf{X}_{m R}\right|\right) /\left|\mathbf{X}_{n L}-\mathbf{X}_{m R}\right|\right]\left|E_{i}\right\rangle\left\langle E_{i}\right|\right\}\right| E_{f}\right\rangle
\end{gathered}
$$

In Eq.(A3b), $k=\sqrt{\left(E_{f}-E_{i}\right)^{2}+a^{-2}} \approx E_{f}-E_{i}$ (if $E_{f}-E_{i}>>a^{-1}$ ) is the wavenumber of a photon making the transition from the excited state to the ground state.

Eq.(A3b) may be compared to Eq. (36c). The gaussian with width $a$ there, is replaced by the $\sin k z / z$ form with width $k^{-1}$ here. An expansion in powers of $a^{-1}$ there is replaced by an expansion in powers of $k$ here. It is because 
$k>>a^{-1}$ that this RCSL model produces a much larger excitation rate than QRCSL. The first non-vanishing term in Eq. $(\mathrm{A} 3 \mathrm{~b})$ is

$$
\Gamma=2 \lambda a k^{5}\left(5 ! \pi^{2}\right)^{-1}\left[\left|\left\langle E_{f}\left|\sum_{n=1}^{N} \mathbf{X}_{n}^{2}\right| E_{i}\right\rangle\right|^{2}+2 \sum_{n=1, m=1}^{N} \sum_{i, j=1}^{3}\left\langle E_{f}\left|\mathbf{X}_{n}^{i} \mathbf{X}_{n}^{j}\right| E_{i}\right\rangle\left\langle E_{i}\left|\mathbf{X}_{m}^{i} \mathbf{X}_{m}^{j}\right| E_{f} \mid\right\rangle\right] .
$$

This is identical to Eq.(37) except for the numerical factor, and is used in Eq.(41).

[1] P. Pearle, Phys. Rev. A 39, 2277 (1989).

[2] G. C. Ghirardi, P. Pearle and A. Rimini, Phys. Rev. A 42, 78 (1990).

[3] P. Pearle, in Sixty-Two Years of Uncertainty, edited by A. Miller, (Plenum, New York 1990), p. 193.

[4] G. C. Ghirardi, R. Grassi and P. Pearle, Found. Phys. 20, 1271 (1990);

[5] P. Pearle in Quantum Chaos-Quantum Measurement, edited by P. Cvitanovic et. al., (Kluwer, the Netherlands 1992), p.283.

[6] P. Pearle, Found. Phys. 30, 1145 (2000).

[7] B. Collett, P. Pearle, F. Avignone and S. Nussinov, Found. Phys. 25, 1399 (1995); P. Pearle, J. Ring, J. I. Collar and F. Avignone, Found. Phys. 29, 465 (1999); G. Jones, P. Pearle and J. Ring, Found. Phys. 34, 1467 (2004).

[8] P. Pearle, Phys. Rev. A 48, 913 (1993); in Stochastic Evolution of Quantum States in Open Systems and in Measurement Processes, edited by L. Diosi and B. Lukacs (World Scientific, Singapore 1994), p. 79; in Perspectives on Quantum Reality, edited by R. Clifton (Kluwer, Dordrecht 1996), p. 93.

[9] A. Bassi and G.C. Ghirardi, Phys. Rev. A65, 042114 (2002).

[10] P. Pearle, Phys. Rev. A59, 80 (1999).

[11] O. Nicrosini and A. Rimini, Found. Phys. 33, 1061 (2003). This manifestly covariant CSL-type model is quasi-relativisitic in that, like QRCSL, the collapse-inducing operator does not commute with itself at spacelike separation. However, it is not quasi-relativistic since, unlike QRCSL, this commutator does not decrease rapidly with increasing distance. This model violates 1), 2), 4) and 5). Although nonrelativistic and Markovian with a nonlocal collapse-inducing operator, with a slight alteration it can be cast as relativistic and non-Markovian with a local collapse-inducing operator. In this form it violates $3), 4)$ and 5).

[12] C. Dove and E. J. Squires, A Local Model of Explicit Wavefunction Collapse, quant-ph /0406094, was the first attempt at a relativistic generalization of GRW's "hitting" process. R. Tumulka, A Relativistic Version of the Ghirardi-Rimini-Weber Model, quant-ph /0406094 has recently presented an ingenious improved model, for free particles satisfying the Dirac equation. It converts free particles to antiparticles, so it violates 5).

[13] G. C. Ghirardi, A. Rimini and T. Weber, Phys. Rev. D 34, 470 (1986); Phys. Rev. D 36, 3287 (1987); Found. Phys. 18, $1,(1988)$.

[14] B. Collett, P. Pearle, F. Avignone and S. Nussinov in reference [7]; B. Collett and P. Pearle, Found. Phys. 33, 1495 (2003).

[15] A. Pais and G. E. Uhlenbeck, Phys. Rev. 79, 145 (1950); G. V. Efimov, JETP 44, 2107 (1963); for a comprehensive discussion and citation of other papers, see K. Namsrai, Nonlocal Quantum Field Theory and Stochastic Quantum Mechanics, (Reidel, Dordrecht, 1986).

[16] P. Pearle and E. Squires, Phys. Rev. Lett. 73, 1 (1994).

[17] National Nuclear Data Center, www.nndc.bnl.gov/index.jsp.

[18] H. S. Miley, F. T. Avignone III, R. L. Brodzinski, J. I. Collar and J. H. Reeves, Phys. Rev. Lett. 65, 3092 (1990).

[19] J. M. Blatt and V. F. Weisskopf, Theoretical Nuclear Physics (Wiley, New York 1952), p.595.

[20] I first heard this suggestion a decade ago from Henry Stapp (private communication), and was amazed that such a distinguished relativisitic quantum field theorist would not be disconcerted if it proved impossible to make a viable relativistic collapse model. 